\title{
Contemplating Potential Chemical Therapeutic Options to Combat Marburg Virus
}

\author{
Umar Saeed ${ }^{1,2 *}$, Zahra Zahid Piracha ${ }^{2,3}$, Aamer Ikram ${ }^{1}$, Rafia Tahir ${ }^{2}$, Rida Fatima ${ }^{2}$, Aniqa Nawaz ${ }^{2}$, \\ Laiqa Shahid², Noor-Ul-Ain², Amna Younus², Ghazala Perveen² and Rijja Hussain ${ }^{4}$ \\ ${ }^{1}$ Biological Production Division, National Institute of Health (NIH), Pakistan \\ ${ }^{2}$ Department of Biological Sciences, International Islamic University, Pakistan \\ ${ }^{3}$ Department of Health Sciences, Health Services Academy (HSA), Pakistan \\ ${ }^{4}$ Department of Bioinformatics, National University of Sciences and Technology (NUST), Pakistan
}

*Corresponding author: Umar Saeed, Biological Production Division, National Institute of Health (NIH), International Islamic University, Pakistan.

To Cite This Article: Umar Saeed, Zahra Zahid Piracha, Aamer Ikram, Rafia Tahir, Rida Fatima, et al., Contemplating Potential Chemical Therapeutic Options to Combat Marburg Virus.AmJ Biomed Sci\&Res. 2021 -13(5). AJBSR.MS.ID.001915. DOI: 10.34297/AJBSR.2021.13.001915.

Received: 眥 July 01, 2021; Published: 䟧 July 30, 2021

\begin{abstract}
Background: Marburg virus (MARV) belongs to Filovirus, which cause deadly hemorrhagic fever in humans. Due to its highly dangerous potential, not much work has been done and its treatment has still to be found yet.

Objective: The aim of this study was to demonstrate potential therapeutic options against MARV. There is no therapy discovered for the treatment of MARV infection, however there are certain therapeutic options, which only enhance the life span of infected ones.

Material and Methods: A literature search was performed by using the keywords; Chemical approaches against MARV, therapeutic options against Marburg, in Pubmed, Bioline International, BioOne, ScienceDirect and Google scholar.

Results: Sixty-five different studies dating from 1994 to May 2021 were included in this study. The activity of certain drugs is dependence upon induction tissue factors and fibrin, while some inhibit cell entry. Several experiments need to be conducted on drugs to examine their potential cure. Current study elaborates up to date chemical approaches as potential therapeutic options to combat Marburg Virus including effects of Protein Inhibitors, Azithromycin, Tamoxifen, Chloroquine, Digitoxin, Tetrandrin, Histamin Receptor, 5HT, Serotonin Receptor, Aminomethyl Benzamide, Monoclonal Antibodies (mAbs), VSV Based Vaccines, Glycoprotein (GP) Pseudoviruses, Interferons (IFNs), G-Protein coupled Receptors, Tyro-3 Receptor Tyrosine Kinase Family, Vaccines with Alpha-Virus Replicons, Inhibitors of ER $\alpha$-glucosidases, Ester Prodrugs of IHVR-19029, Favipiravir,
\end{abstract} Remdesivir, Glycosaminogilycans, Adenovirus Serotype 5 (ADV 5), Ribavirin, and several others.

Conclusion: Although accurate treatment and cure of MARV is not available, however several chemical therapeutic options can be utilized to symptomatically treat the MARV infected patients.

Keywords: Marburg; Filoviruses; MARV; Therapeutic options

Abbreviations: MARV: Marburg Virus; EBOV: Ebola Virus; rVSV: Recombinant Vesicular Stomatitis Virus; LNP: Lipid Nano particles; PMO: Phosphorodiamidate Morpholino Oligomers; GPCR: G-protein coupled Receptors; GAGs: Glycosaminoglycans

\section{Introduction}

Marburg and Ebola viruses both belong to same family of viruses known as Filoviridae (filoviruses). Both of these viruses' species are different structure wise but cause similar hemorrhagic fever in humans and non-human primates. The natural hosts of MARV are fruit bats of Pteropodidae family, known as Rousettus aegyptiacus. The very first cases appeared were transmission of virus from these fruit bats to Humans. Secondary cases were human-to-hu man transmission of viruses through either direct contact or body 
fluids' exchange via blood, body secretions, or innate surfaces like bedding, clothing and several others [1].

Marburg Virus (MARV) causes Marburg virus disease (MVD) in humans, which is quite dangerous and often results in death. Marburg virus cause illness abruptly with high fever, severe headache, muscle aches, watery diarrhea, abdominal cramping, nausea and vomiting and malaise can begin on third day. The patient of this phase has "ghost-like" appearance, deep-set eyes, expressionless faces and extreme lethargy. A non-itchy rash has appeared between 2 and 7 days after the onset of symptoms. Body starts bleeding from multiple areas; fresh blood in vomitus and faeces is accompanied by bleeding from nose, gums and from vagina. Damage to central nervous system can result in confusion, irritability and aggression.

In fatal cases, death usually occurs between 8 and 9 days after onset, due to the severe blood loss and shock [2]. Primary transmission of MARV is still under extensive research and is hard to prevent but its secondary transmission can be avoided by following all general preventive measures used for all other hemorrhagic fevers including EBOV, which includes no direct physical contact with infected person, use of protective mask, gloves, gowns. Infected person should be properly quarantined, needles and other equipment used for such person should be disposed off properly to decrease chances of accidental transmission of infection [1]. The first ever occurrence of Marburg Virus (MARV) was recorded in Marburg and Frankfurt, two cities of Germany and in Belgrade, Serbia in 1967 . The fatality ratio of MARV is up to $88 \%$ with average of $50 \%$ [2]. It has been regarded as Risk Group 4 pathogen s it requires Biosafety Level-4 equivalent containment. This is the main reason not much work has been done regarding its treatment [1].

\section{Protein Inhibitors}

Genstein is a naturally occurring Isoflavonoid phytoestrogen, which is an inhibitor of tyrosine kinase [3]. It is noticed that pre-treatment of host-cells with kinase inhibitor Genestein and tryphostin AG-1478 leads to inhibition or transduction in cells infected with Marburgvirus (filoviruses). It was concluded in results that a mixture consisting of Genestein and Tryphostin AG-1478 which could be labeled as broad-spectrum antivirus is effective against Arenaviruses all species of filoviruses including Marburg Virus in filoviral hemorrhage as therapeutic/prophylactic agent [4].

\section{Azithromycin}

Azithromycin is a common antibacterial and Antiviral use to treat a number of ailments [5]. Although there is no officially recommended drug for treatment of any filoviruses including MARV, however there is a list of FDA approved drugs which have been noticed to be potential candidate for MARV. This has been proved through their combinatorial screening using a pseudo-virion and minigenome replicon systems. One of these drugs is Azithromycin. It is observed to inhibit entry of all types of Filoviruses due to its cationic amphiphilic activity [6].

\section{Tamoxifen}

Tamoxifen belongs to a group of drugs known as anti-estrogens [7]. It has similar mode of action to azithromycin. It allows accumulation of cholesterol to inhibit entry of virus in host-cells. Tamoxifen's cationic amphiphilic structural property allows its sequestration in late endosome/lysosome to interrupt entry of virus [6].

\section{Chloroquine}

Chloroquine belongs to a group of drugs called anti-Malarials [8]. It is usually known to treat malaria and other parasites. For viruses like Ebola and Marburg to invade a host, they need endosomal/ lysosomal acidification so that $\mathrm{pH}$ dependent endosomal proteases can cleave the viral glycoprotein segment to cross the replication process [9]. Without this process, viral replication is not successful. So, drugs which can target this endosomal/lysosomal acidification and its acidic $\mathrm{pH}$ dependent protease can be possible cure for these viral infections [10].

\section{Digitoxin}

Digitoxin is a lipid soluble cardiac glycoside. It inhibits plasma membrane sodium potassium ATPase, resulting in increased level of sodium and calcium inside the cell and decreased levels of potassium [11]. Digitoxin was tested for anti-Ebola virus activity in a BSL-2 lab, using EBOV pseudo-virion and minigenome replicon model. It was reported to potentially block entry of the virus to the cell [6].

\section{Tetrandrin}

Tetrandrin is a naturally occurring, bisbenzylisoquinoline alkaloid extracted from roots of plant known as radix stephania tetrandrae. It non-selectively inhibits activity of calcium channel and also induces G1 blockade in cell cycle's G1 phase and may promote apoptosis [12]. Recent research shows that these alkaloids could be effective against Filoviruses [13]. Filoviruses require endosomal calcium channels two-pore channels (TPCs). Inhibition of these TPCs by genetic or pharmacological approaches, including FDA approved drugs and natural products like tetrandrin prevent Filovirus escape from endosomes [14].

\section{Histamin Receptor, 5HT, Serotonin Receptor}

5 HT receptor is a group of G-protein coupled receptors and ligand gated ion channels found in central and peripheral nervous system. Serotonin receptors regulate the liberation of neurotransmitters such as acetyl-choline, dopamine, epinephrine 
and several others. These inhibitors productive slab the replication of MARV. It plays an analytical role in filoviral entry and can be enlarged as a constructive MARV [15]. Important task of this receptor is to stop Filoviruses in the process of its entry in host cells [16].

\section{Aminomethyl Benzamide}

4-Aminomethyl Benzamide is very efficacious against MARV, as there is no vaccine available for it yet. Through this drug many different compounds and structures can be synthesized like idolines. This 4 -aminomethyl benzamide is very effective to inhibit entry of filoviruses [17].

\section{Monoclonal Antibodies (mAbs)}

Antibodies containing glycoprotein have been shown to provide life-saving therapy in non-human primates. Few monoclonal antibodies (mAbs) show activity against MARV. The most effective mAbs in this panel is GP2 subunit is GP2 subunit that is only present on MARV and not in EBOV and also protect against any lethal exposure up to $90-100 \%$ [18].

\section{VSV Based Vaccines}

An experimental vaccine based on recombinant Vesicular Stomatitis (rVSV) expresses single filovirus glycoprotein (GP) in place of VSV Glycoprotein (G). These rVSV based vaccines have also shown positive results when administered against MARV [18,19]. Neutralizing Antibodies are thought to be one of the best treatment options against MARV. The efficiency of VSV based vaccines express MARV GP or MARV mucin deleted ( $\triangle$ MUC) GP evaluated in BALB/C mice. Results have shown that deleting MLD on vaccine will decrease vaccine efficiency. It would yield more and better neutralizing mAbs than a vaccine expressing full GP [20]. Filovirus vaccine based on recombinant Vesicular Stomatitis Virus (rVSV) that expresses individual filovirus glycoprotein (GP) in place of VSV GP. These vaccines show 100\% efficiency against MARV in nonhuman primates. Animals having anti-MARV GP do not show any signs of clinical disease and all were completely protected against MARV. This shows $100 \%$ protective efficiency against MARV [21].

\section{Glycoprotein (GP) Pseudoviruses}

There is no approved antiviral therapy for treatment of MVD. It supposed that compounds with specific inhibitory activity towards MARV-GP-dependent entry will inhibit the recombinant VSIV-AGMARV-GP pseudotype much more efficiently than control rVSIV$\Delta \mathrm{G}-\mathrm{G}$ pseudotype [22].

\section{Interferons (IFNs)}

Interferons are basically members of protein family that interact with some receptors to activate antiviral defense mechanisms and also promote adaptative immunity. MARV infection not only demonstrated to inhibit IFN $\alpha / \beta$ induced Tyrosine phosphorylation of STAT-I and STAT-II But also to IFN $\Upsilon$-induced STAT phosphorylation. In contrast, MARV VP40 does not detectably inhibit the tyrosine phosphorylation of STAT-II. The mutation of VP40 has no detectable effect on inhibition of IFNs signaling. This shows that MARV inhibit IFN signaling by a different mechanism than other viruses [23].

\section{G-Protein coupled Receptors}

In G-protein coupled Receptors (GPCR) antagonists, Histamin Receptors, 5HT (serotonin) receptors, muscarinic acetylcholine receptor and adrenergic receptor are included. They are inhibitor. They block the replication of MARV, which show that they have vast antiviral activity of (GPCR) antagonists. GPCR antagonists block filoviral (MARV) entry at a step following the initial attachment but before the viral membrane fusion. So GCPR antagonists can be developed as useful MVD therapy [24]. Another GPCR called Antihistamines also show anti-filoviral activity. Anti-histamines are targets for drug repurposing due to their affordable price and easy access mainly because of their vast use. Filovirus entry inhibition is not dependent on cell surface or acetyl-choline receptors but occurs in endosome such as cathepsin inhibitor CS-047. Anti-histamines are best candidate for repurposing anti-filoviral therapeutic and for decrement of unfit histamine or muscarinic receptor interactions without loss of anti-filoviral ability [16].

\section{Tyro-3 Receptor Tyrosine Kinase Family}

Tyro receptor tyrosine kinase family includes Ax1, Dtk and Mer. When these members displace in lymphoid cells, which are highly immune to filoviruses, increase infection by pseudotype viruses which have filoviruse glycoproteinon in their envelope, their infection is reduces by antibodies of Tyro- 3 family members [25]. Effective vaccine for Marburg Virus is not available. To determine possible candidates for MVD, vaccine experiments were performed that either fully inactivated MARV or a Boculovirusexpressed virion subunit could be used to immunize. Guinea pigs against the fatal infection. So irradiated mechanism against MARV. Serum antibodies from immunes were also used against this deadly infection [26]

\section{Vaccines with Alpha-Virus Replicons}

Since irradiated MARV prevents this fatal infection in guinea pigs and RNA replicon which has VEE virus, but when VEE structural genes are replaced by genes of Marburg Glycoproteins, Nucleoproteins, VP40, VP35, VP30 and VP24 were used as vaccines for Guinea Pigs. It shows that GP or NP were protective Antigens while VP35 has incomplete protection. It was also applied on other non-human primates. Its result was that 3 monkeys were inoculated 
with replicon which has MBGV GP, Nad 3 other were inoculated with both replicons that has GP or NP, they all were fully protected from disease [27].

\section{Inhibitors of ER $\alpha$-glucosidases}

In host cells $\alpha$-glucosidases I and II are important for viral glycosylated envelope proteins. Inhibition of glycan processing enzymes causes de-generation of viral glycoproteins and decreases virion secretion. CM-10-18 and imino sugar $\alpha$-glucosidase inhibitor and 3 novel imino sugar decreases the death rate of hemorrhagic fever virus; Marburg [28]. Most functions which are important for viral duplication are targeted and this is considered as broadspectrum antiviral approach. But only some host functions are valid for antiviral approach in-vivo activity. ER $\alpha$-glycosidase I and II are very important for Morphogenesis of some enveloped viruses. Invivo antiviral potential of various ER $\alpha$-glucosidase inhibitors have been reported in animals infected with many viruses such as MARV. Combination of therapeutic strategy imino sugars IH VR-19029 and T-705 for the treatment of viral hemorrhagic fevers has broad spectrum in-host and viral-targeting antiviral agents [29].

\section{Ester Prodrugs of IHVR-19029}

IHVR-19029 is inhibitor of $\alpha$-glucosidases I and II and protect mice from MARV infection and it is administered orally but show low-absorption with gut glucosidases, So, for improvement ester prodrugs are synthesized which are inactive to glucosidases and also have antiviral activities [30].

\section{Favipiravir}

T-705 (favipiravir) is a pyrazinearcarboxamide derivative and it is an antiviral against MARV infection and many other viruses. It is clinically licensed in Japan to treat influenza. So according to studies T-705 is a strong therapeutic agent against MARV [31]. In a study carried out, MARV infected 5 out of 6 animals, 83\% survived when they were treated with Favipirvir while all NHPs which were not treated, died [32].

\section{Remdesivir}

Remdesivir (GS-5734) has therapeutic ability against MARV. Remdesivir treated animals have improved clinical score, low viral RNA and improved markers of Kidney [33]. Remdesivir, basically developed for treatment of MARV and EBOV infections [34]

\section{Role of Glycosaminogilycans}

Heparin along with other glycosaminoglycans (GAGs) can attach to inhibit glycoprotein mediated viral entrance. HS and other GAGS binds to host receptors that are used for infection. GAGS have therapeutic potential and ability to treat the MARV infected patients [35].

\section{Adenovirus Serotype 5 (ADV 5)}

Replication-defective adenovirus serotype 5 (ADV5) vectorbased vaccines has power to induce positive immune responses in the simian-human immunodeficiency virus/rhesus monkey model. One dose of this vaccination of recombinant ADV5 vector builds induced cellular and humoral immunity, but the speedy generation of neutralizing anti-Ad5 antibodies limited the immunity induced by repeated vector administration. The magnitude of the immune responses provoked by this vaccine was greater when they were delivered as boosting immunogens in plasmid DNAprimed monkeys than when they were used as single-modality immunogens. Therefore, ADV5-based vectors in DNA-primed subjects are preferred use of this vaccine modality for generating long-term immune protection [36].

At present, recombinant adenovirus serotype 5 (rAd5; with and without DNA priming) vaccine have undergone the most broad (multiple antigens, multivalent vaccines, cross-protection studies, and so on) testing in non-human primates and consistently demonstrate protection. A number of other platforms do exist (e.g., DNA, other recombinant adenovirus [rAd] platforms, recombinant human parainfluenza virus 3 [rHPIV3] and virus-like particles [VLPs]) but have either not confirmed $100 \%$ efficacious or have not been tested on nonhuman primates.

While DNA prime-rAd5 vaccine regimens have demonstrated to be effective in providing prophylactic defense, but the vaccine dosage schedule requires numerous immunizations over 6 months to 1 year, a time period that is too long to be effective as an intervention during an outbreak or bioterrorism event. Double dose of rAd5 ZEBOV GP provided $100 \%$ protection and viremia was not identified; however, the booster did not enhance antibody response, presumably due to vector immunity through primary immunization.

A single vaccination with only 1012 particles of rAd5 ZEBOV GP and ZEBOV NP also provided $100 \%$ survival in just 28 days post vaccinations. [37] Analysis have shown that at least 1010 particles of rAd5 ZEBOV GP were required to prevent viremia and maintain survival, in addition inclusion of NP was not included [38]. A panfilovirus compound adenovirus (CAdVax) has been developed from four different constructs, delivers two copies of ZEBOV NP and a single copy of MARV-Musoke GP, SEBOV GP, MARV-Musoke NP, ZEBOV GP, MARV-Ci67 GP, MARV-Ravn GP.

Following two vaccinations, this blended vaccine was able to protect cynomolgus macaques from challenge with ZEBOV and MARV-Musoke and subsequent back-challenge with MARV-Ci67 and SEBOV respectively [21]. Animals showed no signs of illness in liver enzymes or comparable IgG titers against all five filoviruses after vaccinations. This showed that a single vaccine can provide 
protection against multiple filoviruses. Likewise, a multivalent vaccine candidate, which expresses ZEBOV GP and SEBOV GP in a single CAdVax, was able to provide protection against parenteral and aerosol challenge with SEBOV (this required two vaccinations) and ZEBOV [39].

Pre-existing protection to Ad5 (present up to $60 \%$ of the general population and as high as $85 \%$ in Africa) is critical for the vaccine efficacy, like macaques with immunity to Ad5 were not protected from MARV challenge, when immunized with rAd5ZEBOV GP [40]. This problem is solved by using different serotypes of adenovirus. A chimpanzee-based adenovirus vaccine (AdC7) showing ZEBOV GP stimulated robust T- and B-cell responses against ZEBOV in naive mice, inducing $100 \%$ protection from homologous lethal challenge [41]. This has the ability to protect $100 \%$ of guinea pigs from disease, with low vaccine dose of $5 \times 109$ particles/kg. Preexisting protection to Ad5 in mice did not affect efficacy of vaccine, suggesting it has ability to be used to circumvent pre-existing immunity. A chimeric simian adenovirus AdC5/C1 expressing ZEBOV GP was also generated (AdC5/C1 ZEBOV GP), as it induced ZEBOV GP-specific T- and B-cell responses. These were of minor magnitude as compared to rAd5 constructs; however, the entire IgG response to ZEBOV GP was comparable. A single dose of AdC5/C1 ZEBOV protected mice from lethal challenge [42]. Rhesus macaques vaccinated with AdC5/ C1 ZEBOV GP mounted T-cell and antibody responses to the ZEBOV GP. Possibly it might be a better strategy to prevent pre-existing immunity, however it requires experimentation in nonhuman primate animal model. As an alternative means of avoiding pre-existing immunity, Ad-based vectors can be delivered by nasal vaccination, which protects against lethal challenge in ZEBOV mouse model.

Both $\mathrm{T}$ - and B-cell responses observed in mice receiving nasal vaccination indicate a qualitative improvement of immune response after mucosal immunization in contrast to intramuscular vaccination. Therefore, mucosal immunization consequences in better stimulation of CD8+ T cells and effectors memory T cells than intramuscular administration of the vaccine [43]. But, presence of pre-existing immunity, only mice vaccinated by intranasal route was protected [44].

An advanced formula of rAd5 vector that contains an improved expression cassette (Ad-CAGoptZGP) showed higher expression of ZEBOV GP and significantly improved T- and B-cell responses at doses that were 10 to 100 -fold lesser than that compulsory with Ad-CMVZGP. This vaccine provided $100 \%$ protection against this disease in mice at a dose 100-times lower than the previous rAd5 vector. Luckily, it was able to protect mice when given $30 \mathrm{~min}$ post challenge, suggesting that rAd-based vectors may also have the ability to provide protection post exposure [45]. While these data are exciting, it must be demonstrated in the nonhuman primate, where it has proven more problematic to protect post exposure when compared with either the mouse or guinea pig models.

\section{Recombinant VSV}

Vesicular stomatitis virus, a member of the Rhabdoviridae family, has been transformed into an attenuated replication-competent recombinant vaccine (rVSV) for many pathogens including HIV, hepatitis B virus, influenza, SARS coronavirus, respiratory syncytial virus, human papillomavirus (HPV) and HPV-associated cancer. The native VSV G was replaced by MARV glycoprotein to produce surface expressing MARV GP, VSV like particles. In the beginning of the study, both rVSV-MARV GP and rVSVZEBOV GP demonstrated $100 \%$ protection against homologous challenge in cynomolgus macaques following a single intramuscular immunization [46]. Animals vaccinated with the vaccine rVSVZEBOV GP that remained alive challenge were back-challenged with SEBOV. All showed symptoms and only one out of four remain alive. The survivor may be credited to the SEBOV not resulting in $100 \%$ lethality [47]. rVSVMARV GP vaccinated animals survived with no symptoms. Crossprotection between heterologous MARV strains has also been verified in cynomolgus macaques that were saved from challenge strains Ravn (the most divergent MARV isolate) and Angola (the most virulent MARV isolate to date) when vaccinated foe only 28 days prior with rVSV-MARV GP (strain Musoke) [48]. Several routes of immunization with rVSV have also been demonstrated to be very effective. Delivering the rVSV-ZEBOV GP vaccine by the oral, intranasal or intramuscular route for 28 days protected all cynomolgus macaques from subsequent MARV challenge [49]. None of the vaccinated animals developed IgG titers against MARV by the time of MARV challenge; however, IgG was detectable following infection in three animals. This suggests that the short-lived lowlevel VSV viremia may be essential for protection, CD4+ cells play a role in protection and IgG antibodies may not be as essential for protection.

\section{Ribavirin}

The antiviral efficiency of the peptidomimetic furin inhibitor MI-701 in blend with oseltamivir carboxylate and ribavirin against the Marburg virus that are activated by the host protease furin. Cell cultures infected with the strains A/Thailand/1(KAN-1)/2004 (H5N1) and A/FPV/Rostock/1934 (H7N1) were treated with each agent single, or in double and triple mixtures. MI-701 alone achieved a concentration-dependent decrease the virus propagation. MI-701 treatment with oseltamivir carboxylate or with triple combination with ribavirin showed viral inhibition. MI-701 resistant mutants were not experimented. Emergence of NA mutation $\mathrm{H} 275 \mathrm{Y}$ conferring high oseltamivir resistance was delayed in the presence of MI-701 [50]. The drugs desferal, ribavirin and homologous 
(guinea pig) interferon was experimented on Marburg fever Ribavirin is very effective. A moderate response to desferal was noted. It can be inferred that TNF can cause Marburg hemorrhagic fever.

\section{Lipid encapsulated small infecting RNA (SiRNA)}

Lipid nanoparticles (LNP) delivery of anti-MARV nucleoprotein targeting small interfering RNA is very effective for Marburg virus. Twenty-one rhesus monkeys were challenged with a lethal dose of Marv-Angola.16 of these animals were treated with LNP containing anti-MARV NP siRNA beginning at 30 to $45 \mathrm{~min}$, after 1 day or 2 day after the virus challenge. The disease was significantly prevented in macaques treated with anti-MARV NP siRNA (LNP-encapsulated), which demonstrates its possible therapeutic option for treatment against MARV in humans [51]. Treatment shows recovery of about $60 \%-100 \%$ survival of guinea pigs infected with MARV. Although treatment with siRNA targeting other MARV messenger RNA (mRNA) had a beneficial impact, targeting the MARV NP mRNA resulted in the highest survival rates. NP-718m siRNA in lipid nanoparticles provided $100 \%$ protection against MARV strains Angola and Ci67, and 60\% against Ravn. A cocktail containing NP$718 \mathrm{~m}$ and NP-143m provided 100\% defense against MARV Ravn [52].

\section{AVI-6002 \& AVI-6003}

The development of antisense Phosphorodiamidate Morpholino Oligomers (PMOs) AVI-6002 (composed of AVI-7357 and AVI7539) and AVI-6003 (composed of AVI-7287 and AVI-7288) have proved much effective targeting Ebola virus and Marburg virus respectively. Detection process involved identification of optimal transcript binding sites for PMO based RNA-therapeutics followed by screening for effective viral gene target in mouse and guinea pig models utilizing adapted viral isolates. Phosphorodiamidate Morpholino Oligomers (PMO) were modified by conjugating with cell penetrating peptide, and positively charged linkages, the two agents which target separate genes. In the last analysis, a single agent for treatment of each virus was selected, AVI-7537 targeting the VP24 gene of Ebola virus and AVI-7288 targeting NP of Marburg virus, and are now progressing into late-stage clinical development as the optimal therapeutic candidates [53].

\section{Bornyl ester derivatives containing saturated $\mathrm{N}$-heterocycles}

The bornyl ester derivatives containing saturated N-heterocycles showed the highest antiviral activity. It is supposed that elements with specific inhibitory activity toward MarV-GP-dependent virus entry will hinder the rVSIV- $\Delta$ G-MarV-GP pseudotype much more accurately than the control rVSIV- $\Delta$ G-G pseudotype. At the same period, the compounds similarly inhibiting both pseudotypes will likely affect rVSIV capsid replication common to the entry of both viruses. Borneol itself is not active against both pseudotypes and is not harmful, whereas its derivatives have varying toxicity and antiviral activity. Six of the borneol derivatives with low-toxicity were inhibitors of MARV-GP-infection. Of them, compound 6 containing a methylpiperidine moiety exhibited the highest virusspecific activity [54].

\section{Calcium Channel Blocker (Bepridil)}

For the treatment of filovirus infection until no therapeutics are approved. For treating angina, a calcium channel blocker called as bepridil was developed and recognized as effective inhibitor of filovirus invitro (Ebola \& Marburg virus) and in vivo (Ebola). Effectuality of bepridil in lethal mouse model of Marburg virus disease was evaluated. $80 \%$ or $90 \%$ survival results were noted when $12 \mathrm{mg} / \mathrm{kg}$ bepridil, once or twice given to them daily, respectively. This data shows anti filovirus activity of bepridil and further investigation of bepridil was warranted, by similar mechanisms, as a pan-filo virus therapeutic agent [55].

\section{Nematode anticoagulant protein C2 (rNAPC2)}

Procoagulant tissue factor TF seems to play a part in coagulation disorder that indicates filo-virus infection. In rhesus macaques, along with Angola strain of Marburg virus (MARV-Ang) pathogenesis of lethal infection was evaluated. And as a potential treatment affectivity of recombinant nematode anticoagulant protein c2 (rNAPc2) an inhibitor of TF/factor VII-a, was tested. With the high dose of approximately (1000 pfu) of MARV-Ang 12 rhesus macaques were challenged. Six of them were served as a control animal and six of them were treated with rNAPc2. As a result, all six control animals challenged with MAR-Ang, died by day 8 or we can say 7.3 days. However, animals treated with rNAPc2 5 of 6 died on ninth day and one of them survived. MARV-Ang infection course seemed to be progress more quickly in rhesus macaques than other strains of MARV, which was reported earlier. In macaque up-regulation of TF was not as striking in contrast to Ebola virus.

Deposition of fibrin was not too much prominent pathologic characteristics of disease in these animals. From this data, we are able to know that disease causes ability of MARV-Ang infection seems to be compatible with enhanced virulence of human attributed to strain. The less effectivity of rNAPc2 against MARVAng infection, as compared to its usefulness against EBOV, it seems to be dependent on difference in TF induction and fibrin [56].

Recombinant nematode anticoagulant protein C2 (rNAPC2) have a result, that is survival of 3 of 9 treated and infected rhesus monkeys [57]. A human activated protein C (rhAPC) was also in some extends effective in providing survival of 2 of 11 infected and treated rhesus monkeys. Viral gene-specific therapies were involved in the survival benefit that was observed in infected nonhuman primate lethal challenges model [58]. 


\section{Immunosmodifier; ridostin, reaferon, polyribonate}

In an experiment, guinea pigs infected with Venezuelan, equine, encephalomyelitis (VEE) (strain Trinidad), Marburg (strain popp) and Ebola (M/C-8 variant of Zaire strain) try viruses were given 5 to 20 respiratory LD50 doses of immunomodifier ridostin,reaferon and Polyribonate. Therapeutic effects and polyphlactic effects of these drugs were used alone or in combination. Animals infected with Marburg virus $(\mathrm{p}=0.1)$ are protected by urgent prophylactic simultaneous intramuscular an intranasal administration of ridostin and prolonged their lifespan by 2.4 days $(p=0.1$ Only the lifespan of these infected animals are prolonged by the use of these drugs. In any of the studied infection, all of these drugs did not give satisfied therapeutic effects [59].

\section{Amiodarone, drone-darone and verapamil}

Depending upon various cellular factors filovirus use complex route of cell entry. It is hypothesized that the drugs that interfere with signal transduction and cellular process required for their entry are already accepted for human use for other indications and have a anti filovirus properties. Authentic filoviruses and lentiviral particles pseudo typed with filoviral glycoprotein was used to recognized and categorized these compounds. Amiodarone, a multiion channel inhibitor and adrenoceptor antagonist, is an effective inhibitor of filovirus cell entry at concentrations that reached on daily basis in human serum during anti-arrhythmic therapy, was discovered. It was observed that Amiodarone-related agent dronedarone and the L-type calcium channel blocker verapamil have similar effects.

Pseudo viruses as well as authentic filoviruses are similarly affected by inhibition amiodarone and they are concentration dependent. Observance of Inhibition of filovirus entry was with most but not all cell types that was tested and was emphasized by the pre-treatment of cells, which indicates a host cell-directed mechanism of action. It is concluded that inhibition of filo viral cell entry is due to the ion channel blockers amiodarone, dronedarone and verapamil [60]. Recombinant nematode anticoagulant protein C2 (rNAPC2) have a result, that is survival of 3 of 9 treated and infected rhesus monkeys. A human activated protein C (rhAPC) was also in some extend effective in providing survival of 2 of 11 infected and treated rhesus monkeys [61]. Viral gene-specific therapies were involved in the survival benefit that was observed in infected nonhuman primate lethal challenges model [62].

\section{AIV-7288}

AVI-7288 is a phosphorodiamidate morpholino oligomer having positive charges, viral messenger RNA that encodes Marburg virus (MARV) nucleoprotein is its target. Its safety in humans is disputable. The efficiency of AVI-7288 in various studies involving a lethal challenge with MARV in nonhuman primates was assessed. Based upon pharmacokinetics in nonhuman primates, dose efficacy was determined. In this method14 once-daily infusions of AVI-7288 (1 mg, $4 \mathrm{mg}, 8 \mathrm{mg}, 12 \mathrm{mg}$, or $16 \mathrm{mg}$ per kilogram of body weight) or in a 3:1 ratio was given to 40 healthy humans ( 8 humans per dose group).

In this type of randomized, multiple-ascending-dose study, safety of AVI-7288 was evaluated. In infected nonhuman primates' survival was dose dependent. Hence, monkeys treated with 0 $\mathrm{mg}, 3.75 \mathrm{mg}, 7.5 \mathrm{mg}, 15 \mathrm{mg}, 20 \mathrm{mg}$, and $30 \mathrm{mg}$ of AVI-7288 per kilogram, have survival rate of $0 \%, 30 \%, 59 \%, 87 \%, 100 \%$, and $100 \%$ respectively. No safety concern was recognized at Doses up to 16 milligram per kilogram per day in humans. Serious adverse event was not reported. In both nonhuman primates and humans, drug exposure was dose dependent, however drug clearance was independent of dose but has higher ratio in nonhuman primates than in humans. on the basis of exposure, $9.6 \mathrm{mg}$ per kilogram per day (95\% confidence interval, 6.6 to 12.5 ) for 14 days is protective dose in humans, that was estimated initially. Someone simulations supported a dose of $11 \mathrm{mg}$ per kilogram per day was protective exposure in nonhuman primates. It was concluded that AVI-7288 has efficiency of post exposure prophylaxis for MARV infection in humans. It happened on the basis of efficacy in nonhuman primates and pharmacokinetic data in humans [63].

\section{Anti vp35 antibody}

Satisfied, effective, and safe therapeutic or prophylactic counter measures are not frequently available. Phage display was addressed to engineer a synthetic antibody, sFab H3, which binds with the Marburg virus VP35 protein (mVP35) with specificity. mVP35 is a condemnatory cofactor of the viral replication complex and a work as a immune antagonist for virus. sFab H3 showed high specificity for mVP35, which is not for Ebola virus VP35. sFab H3 repressed viral-RNA synthesis in a mini genome analysis, encouraging its effective use as an antiviral. We designate sFab H3 by a combination of biophysical and biochemical methods and a crystal shape of the complex. $1.7 \AA$ A resolution defined the molecular dealing between the sFab H3 and mVP35 interferon inhibitory preserve. Study recognizes mVP35 as a therapeutic target using a focus on, that gave a framework for producing engineered Fabs who target other viral protein [64].

\section{rMARV EGFP}

The triggering of recombinant intensify green fluorescent protein (EGFP)-expressing viruses has considerably improved the study of their life cycle and established the possibility for the rapid screening of antiviral drugs. EGFP expressing recombinant MARV from ATU transcription unit was reported. There was insertion of ATU between the second and third genes, encoding VP35 and VP40, 
respectively. Live-cell imaging was used to abide by virus spread in real time. There was detection of EGFP expression at 32 hours postinfection (hpi), and infection of surrounding cells was supervised at $55 \mathrm{hpi}$. As compare to parental virus, production of progeny rMARVEGFP was decreased 4-fold and protein levels of VP40 became low, but not nucleoprotein, were observed, expressing a reduction in downstream protein expression due to the ATU insertion. Interestingly, EGFP have high concentration in viral incorporation in infected cells. This was again produced by transient expression of both EGFP and other fluorescent proteins across with nucleocapsid proteins of filovirus and may instruct that a general increase in protein synthesis happens at viral incorporation sites. Therefore, it is concluded that MARV expressing EGFP could be a critical strategy to prevent viral propagation and screening for antiviral compounds, and exploring molecular mechanisms of inclusion body formation [65].

\section{Conclusion}

There is no therapy discovered for the treatment of Marburg virus infection, however there are some drugs, which only enhance the life span of infected animal. Several drugs have been identified to inhibit filo virus cell entry or replication. Although there are such drugs which did not treat Marburg virus infection, but may warrant possible ways for further investigation. Experiments have been conducted on aforementioned drugs to find its potential cure.

\section{Acknowledgment}

We would like to acknowledge the staff of BPD NIH and IIUI Islamabad for their cooperation and support.

\section{Conflict Of Interest}

Authors have no conflict of interests.

\section{Authors Contribution}

US is principal investigator of the study. US Conceived the study, wrote the manuscript and critically reviewed the manuscript. ZZP, RT, RF, AN, LS, NUA, AY, GP, and RH performed the literature search and assisted US in manuscript writing. AI finally approved the study.

\section{References}

1. Centers for Disease Control and Prevention (2019) Marburg Virus 2020 India.

2. World Health Organization (2018) Marburg Virus 2020 Switzerland.

3. Spinozzi F, Pagliacci M C, Migliorati G, Moraca R, Grignani F, et.al. (1994) The natural tyrosine kinase inhibitor genistein produces cell cycle arrest and apoptosis in Jurkat T-leukemia cells. Leukemia research 18(6): 431439.

4. Kolokoltsov A A, Adhikary S, Garver J, Johnson L, Davey R A, et.al. (2012) Inhibition of Lassa virus and Ebola virus infection in host cells treated with the kinase inhibitors genistein and tyrphostin. Archives of virology 157(1): 121-127.
5. National Health Service (2019) Azithromycin 2020 Britain

6. Du X, Zuo X, Meng F, Wu F, Zhao X, et.al. (2020) Combinatorial screening of a panel of FDA-approved drugs identifies several candidates with antiEbola activities. Biochemical and biophysical research communications 522(4): 862-868.

7. (2020) Tamoxifen, Oral Tablet. Healthline Tamoxifen.

8. (2020) Chloroquine. Webmd Drugs \& Medications.

9. Kissing S, Hermsen C, Repnik U, Nesset C K, Von Bargen (2015) Vacuolar ATPase in phagosome-lysosome fusion. Journal of Biological Chemistry 290(22): 14166-14180.

10. Al Bari M A A (2017) Targeting endosomal acidification by chloroquine analogs as a promising strategy for the treatment of emerging viral diseases. Pharmacology research \& perspectives 5(1): 0023.

11.(2020) National Center for Biotechnology Information. PubChem Compound Summary for CID 441207, Digitoxin.

12. Bauta WE, McDonough JA, Mahindaratne MP, Davey RA (2016) U.S. Patent Application No. 15/078,782.

13. Sakurai Y, Kolokoltsov A A, Chen C C, Tidwell M W, Bauta W E, et.al. (2015) Two-pore channels control Ebola virus host cell entry and are drug targets for disease treatment. Science 347(6225): 995-998.

14. Feldmann F, Sanchez A, Geisbert JB (2013) Filoviridae: Marburg andEbola viruses. In: Knipe DM, et al. (Eds.), Fields virology (6th edn), vol II, Wolters Kluwer Lippincott Willliams \& Wilkins, USA, pp. 923-956.

15. Cheng H, Lear Rooney C M, Johansen L, Varhegyi E, Chen Z W, et.al. (2015) Inhibition of Ebola and Marburg virus entry by G protein-coupled receptor antagonists. Journal of Virology 89(19): 9932-9938.

16. Gaisina I N, Peet N P, Wong L, Schafer A M, Cheng H, et.al. (2020) Discovery and Structural Optimization of 4-(Aminomethyl) benzamides as Potent Entry Inhibitors of Ebola and Marburg Virus Infections. Journal of Medicinal Chemistry 63(13): 7211-7225.

17. Geisbert T W, Daddario DiCaprio K M, Geisbert J B, Reed D S, Feldmann F, et.al. (2008) Vesicular stomatitis virus-based vaccines protect nonhuman primates against aerosol challenge with Ebola and Marburg viruses. Vaccine 26(52): 6894-6900.

18. Geisbert T W, Feldmann H (2011) Recombinant vesicular stomatitis virus-based vaccines against Ebola and Marburg virus infections. The Journal of infectious diseases 204(suppl_3): 1075-1081.

19. Cabral T M, Baig A, Berhane Y,Schmidt L, Hole K, et.al. (2014) Development of neutralizing monoclonal antibodies against the pandemic H1N1 virus (2009) using plasmid DNA immunogen. J Virol Methods 195: 54-62.

20. Mire C E, Geisbert J B, Agans K N, Satterfield B A, Versteeg K M, et.al. (2014) Durability of a vesicular stomatitis virus-based marburg virus vaccine in nonhuman primates. PloS one 9(4): 94355.

21. Kononova A A, Sokolova A S, Cheresiz S V, Yarovaya O I, Nikitina R A et.al. (2017) N-Heterocyclic borneol derivatives as inhibitors of Marburg virus glycoprotein-mediated VSIV pseudotype entry. MedChemComm 8(12): 2233-2237.

22. Valmas C, Grosch M N, Schümann M, Olejnik J, Martinez O (2010) Marburg virus evades interferon responses by a mechanism distinct from ebola virus. PLoS Pathog 6(1): 1000721.

23. Cheng H, Lear Rooney C M, Johansen L, Varhegyi E, Chen Z W, et.al. (2015) Inhibition of Ebola and Marburg virus entry by G protein-coupled receptor antagonists. Journal of virology 89(19): 9932-9938.

24. Shimojima M, Takada A, Ebihara H, Neumann G, Fujioka K, et.al. (2006) Tyro3 family-mediated cell entry of Ebola and Marburg viruses. Journal of Virology 80(20): 10109-10116. 
25. Hevey M, Negley D, Pushko P, Smith J, Schmaljohn A (1998) Marburg virus vaccines based upon alphavirus replicons protect guinea pigs and nonhuman primates. Virology 251(1): 28-37.

26. Chang J, Warren TK, Zhao X, Gill T, Guo F, et.al. (2013) Small molecule inhibitors of ER $\alpha$-glucosidases are active against multiple hemorrhagic fever viruses. Antiviral Res 98(3): 432-440.

27. Ma J, Zhang X, Soloveva V, Warren T, Guo F, et.al. (2018) Enhancing the antiviral potency of ER $\alpha$-glucosidase inhibitor IHVR-19029 against hemorrhagic fever viruses in vitro and in vivo. Antiviral Res 150:112122.

28. Ma J, Wu S, Zhang X, Guo F, Yang K, et.al. (2017) Ester Prodrugs of IHVR19029 with Enhanced Oral Exposure and Prevention of Gastrointestinal Glucosidase Interaction. ACS medicinal chemistry letters 8(2): 157-162.

29. Zhu W, Zhang Z, He S, Wong G, Banadyga L, et al. (2018) Successful treatment of Marburg virus with orally administrated T-705 (Favipiravir) in a mouse model. Antiviral research 151: 39-49.

30. Sandra L Bixler, Thomas M Bocan, Jay Wells, Kelly S Wetzel, Sean A Van Tongeren, et al. (2018) Efficacy of favipiravir (T-705) in nonhuman primates infected with Ebola virus or Marburg virus. Antiviral res 151: 97-104.

31. Danielle P Porter, Jessica M Weidner, Laura Gomba, Roy Bannister, Christiana Blair, et al. (2020) Remdesivir (GS-5734) Is Efficacious in Cynomolgus Macaques Infected With Marburg Virus. J Infec Dis 222(11) 1894-1901.

32. Kamal Kant Sahu, Ajay Kumar Mishra, Amos Lal (2020) COVID-2019: update on epidemiology, disease spread and management. Monaldi arch chest Dis 90(1): 10.4081

33. Aileen O Hearn, Minxiu Wang, Han Cheng, Calli M Lear Rooney, Katie Koning (2015) Role of EXT1 and Glycosaminoglycans in the Early Stage of Filovirus Entry. Journal of virology 89(10): 5441-5449.

34. Sampa Santra 1, Michael S Seaman, Ling Xu, Dan H Barouch, Carol I Lord, et al. (2005) Replication-defective adenovirus serotype 5 vectors elicit durable cellular and humoral immune responses in nonhuman primates. J virol 79(10): 6516-6522.

35. Nancy J Sullivan, Thomas W Geisbert, Joan B Geisbert, Ling Xu, Zhi Yong Yang, et al. (2003) Accelerated vaccination for Ebola virus haemorrhagic fever in non-human primates. Nature 424(6949): 681-684.

36. Nancy J Sullivan, Thomas W Geisbert, Joan B Geisbert, Devon J Shedlock, Ling Xu, et al. (2006) Immune protection of nonhuman primates against Ebola virus with single low-dose adenovirus vectors encoding modified GPs. PLoS Med 3(6): 177.

37. Dana L Swenson, Danher Wang, Min Luo, Kelly L Warfield, Jan Woraratanadharm, et al. (2008) Vaccine to confer to nonhuman primates complete protection against multistrain Ebola and Marburg virus infections. Clin vaccine immunol 15(3): 460-467.

38. William D Pratt, Danher Wang, Donald K Nichols, Min Luo, Jan Woraratanadharm, et al. (2010) Protection of nonhuman primates against two species of Ebola virus infection with a single complex adenovirus vector. Clin vaccine immunol 17(4): 572-581.

39. Thomas W Geisbert, Daniel G Bausch, Heinz Feldmann (2010) Prospects for immunisation against Marburg and Ebola viruses. Rev Med Virol 20(6): 344-357.

40. Gary P Kobinger, Heinz Feldmann, Yan Zhi, Gregory Schumer, Guangping Gao, et al. (2006) Chimpanzee adenovirus vaccine protects against Zaire Ebola virus. Virology 346(2): 394-401

41. Soumitra Roy, Yan Zhi, Gary P Kobinger, Joanita Figueredo, Roberto Calcedo, et al. (2006) Generation of an adenoviral vaccine vector based on simian adenovirus 21. J Gen virol 87(9): 2477-2485.
42. Ami Patel, Yi Zhang, Maria Croyle, Kaylie Tran, Michael Gray, et al. (2007) Mucosal delivery of adenovirus-based vaccine protects against Ebola virus infection in mice. J Infect Dis 196(Suppl. 2): 413-420.

43. Maria A Croyle, Ami Patel, Kaylie N Tran, Michael Gray, Yi Zhang, et al. (2008) Nasal delivery of an adenovirus-based vaccine bypasses preexisting immunity to the vaccine carrier and improves the immune response in mice. PloS one 3(10): 3548

44. Jason S Richardson, Michel K Yao, Kaylie N Tran, Maria A Croyle, James E Strong, et al. (2003) Enhanced protection against Ebola virus mediated by an improved adenovirus-based vaccine. PloS one 4(4): 5308.

45. Steven M Jones, Heinz Feldmann, Ute Ströher, Joan B Geisbert, Lisa Fernando, et al. (2005). Live attenuated recombinant vaccine protects nonhuman primates against Ebola and Marburg viruses. Nat med 11(7): 786-790.

46. Douglas S Reed, Mansour Mohamadzadeh (2007) Status and challenges of filovirus vaccines. Vaccine 25(11): 1923-1934.

47. Kathleen M Daddario DiCaprio, Thomas W Geisbert, Joan B Geisbert, Ute Ströher, Lisa E Hensley, et al. (2006) Cross-protection against Marburg virus strains by using a live, attenuated recombinant vaccine. J virol 80(19): 9659-9666

48. Thomas W Geisbert, Joan B Geisbert, Anders Leung, Kathleen M Daddario DiCaprio, Lisa E Hensley, et al. (2009) Single-injection vaccine protects nonhuman primates against infection with Marburg virus and three species of Ebola virus. J virol 83(14): 7296-7304

49. Yinghui Lu, Kornelia Hardes, Sven O Dahms, Eva Böttcher Friebertshäuser, Torsten Steinmetzer, et al. (2015) Peptidomimetic furin inhibitor MI701 in combination with oseltamivir and ribavirin efficiently blocks propagation of highly pathogenic avian influenza viruses and delays high level oseltamivir resistance in MDCK cells. Antiviral res 120: 89100 .

50. Emily P Thi, Chad E Mire, Raul Ursic Bedoya, Joan B Geisbert, Amy C H Lee, et al. (2014) Marburg virus infection in nonhuman primates: Therapeutic treatment by lipid-encapsulated siRNA. Sci trans med 6(250): 250ra116-250ra116

51. Raul Ursic Bedoya, Chad E Mire, Marjorie Robbins, Joan B Geisbert, Adam Judge, et al. (2014) Protection against lethal Marburg virus infection mediated by lipid encapsulated 209(4): 562-570.

52. Patrick L Iversen, Travis K Warren, Jay B Wells, Nicole L Garza, Dan V Mourich, et al. (2012) Discovery and early development of AVI-7537 and AVI-7288 for the treatment of Ebola virus and Marburg virus infections. Viruses 4(11), 2806-2830.

53. A A Kononova, A S Sokolova, S V Cheresiz, O I Yarovaya, R A Nikitina, et al. (2017) N-Heterocyclic borneol derivatives as inhibitors of Marburg virus glycoprotein-mediated VSIV pseudotype entry. MedChemComm, 8(12): 2233-2237.

54. Lisa Evans DeWald, Julie Dyall, Jennifer M Sword, Lisa Torzewski, Huanying Zhou et al. (2018) The calcium channel blocker bepridil demonstrates efficacy in the murine model of Marburg virus disease. J infect dis 218(suppl_5): S588-S591.

55. Thomas W Geisbert, Kathleen M Daddario DiCaprio, Joan B Geisbert, Howard A Young, Pierre Formenty, et al. (2007) Marburg virus Angola infection of rhesus macaques: pathogenesis and treatment with recombinant nematode anticoagulant protein $\mathrm{c} 2$. J infect dis 196(Supplement_2): 372-S381.

56. Amy C Shurtleff, Travis K Warren, Sina Bavari (2011) Nonhuman primates as models for the discovery and development of ebolavirus therapeutics. Expert Opin Drug Discov 6(3): 233-250.

57. Lisa E Hensley, Edward L Stevens, S Betty Yan, Joan B Geisbert, William L Macias, et al. (2007) Recombinant human activated protein C for 
the postexposure treatment of Ebola hemorrhagic fever. J infect dis 196(Supplement_2): 390-399.

58. Thomas W Geisbert, Amy C H Lee, Marjorie Robbins, Joan B Geisbert, Anna N Honko, et al. (2010) Postexposure protection of non-human primates against a lethal Ebola virus challenge with RNA interference: a proof-of-concept study. Lancet 375(9729): 1896-1905.

59. A N Sergeev, A B Ryzhikov, L E Bulychev, N K Evtin, O V Piankov, et al (1997) Study of the treatment-prophylactic effect of immunomodulators in experimental infections, caused by Marburg, Ebola, and Venezuelan equine encephalitis viruses. Vopr Virusol 42(5): 226-229.

60. Lisa E Hensley, Edward L Stevens, S Betty Yan, Joan B Geisbert, William L Macias, et al. (2007) Recombinant human activated protein $C$ for the postexposure treatment of Ebola hemorrhagic fever. J Infect Dis 196 Suppl 2: 390-399.
61. Gerrit Gehring, Katrin Rohrmann, Nkacheh Atenchong, Eva Mittler, Stephan Becker, et al. (2014) The clinically approved drugs amiodarone, dronedarone and verapamil inhibit filovirus cell entry. J Antimicrob Chemother 69(8): 2123-2131.

62. Alison E Heald, Jay S Charleston, Patrick L Iversen, Travis K Warren, Jay B Saoud, et al. (2015) AVI-7288 for Marburg virus in nonhuman primates and humans. N Engl J Med 373(4): 339-348.

63. Parmeshwar Amatya, Nicole Wagner, Gang Chen, Priya Luthra, Liuqing Shi, et al. (2019) Inhibition of Marburg Virus RNA Synthesis by a Synthetic Anti-VP35 Antibody. ACS infect dis 5(8): 1385-1396.

64. Kristina Maria Schmidt, Michael Schümann, Judith Olejnik, Verena Krähling, Elke Mühlberger (2011) Recombinant Marburg virus expressing EGFP allows rapid screening of virus growth and real-time visualization of virus spread. J infect dis 204(suppl_3): 861-870. 